\title{
High Speed Compressible Turbulence With/without Combustion: Fundamental Understandings and Challenges
}

\author{
Qinling Li \\ Material and Engineering Research Institute, Department of Engineering and Mathematics \\ Sheffield Hallam University \\ Sheffield S1 1WB, UK \\ Q.Li@shu.ac.uk
}

Compressible turbulence is fundamental which arises in various industrial applications. Many regimes have been widely studied by researchers. In this keynote lecture, different CFD approaches will be reviewed. First of all, high-order direct numerical simulation (DNS) of fundamental compressible wall-bounded turbulence will be presented, such as fully developed compressible channel flow and oblique shock waves/turbulent boundary layer interactions. The CFD method is applicable to general geometries. It contains no upwinding, artificial dissipation, or filtering. Instead the method relies on the stabilizing mechanisms of an appropriate conditioning of the governing equations and the use of compatible spatial difference operators for the interior points (interior scheme) as well as the boundary points (boundary scheme). An entropy-splitting approach splits the inviscid flux derivatives into conservative and nonconservative portions. The spatial difference operators satisfy a summation-by-parts condition, leading to a stable scheme for the initial boundary value problem using a generalized energy estimate. A Laplacian formulation of the viscous and heat conduction terms on the right hand side of the NavierStokes equations is used to ensure that any tendency to odd-even decoupling associated with central schemes can be countered by the fluid viscosity. The resulting methods are able to minimize the spurious high-frequency oscillations associated with pure central schemes, especially for long time integration applications. The spatially developing boundary layer is generated using an idealized unsteady analytic inflow profile that emulates the dynamical features of wall-bounded turbulence; this approach has the advantage of creating a self-contained simulation with deterministic inflow conditions that prompt the realistic transfer of energy from the mean flow to the turbulence, and thereby a realistic fully developed turbulent boundary layer in a fairly short downstream distance. It is demonstrated that the methods are robust in terms of grid resolution, and in good agreement with previous published data. The second part of the lecture will focus on various industrial applications by employing RANS and LES methods, with structured and unstructured meshes. Resulting accuracies and errors, such as mesh quality/type, truncation error, turbulence model error, etc. will be addressed. Compressible wallbounded and free shear flows, jet in crossflow with/without shock-waves, shock trains, turbulence mixing \& enhancement, and combustion mechanism will be discussed.

Keywords: Compressibility effects, wall-bounded and free shear flows, numerical accuracy and stability, entropy splitting, higher order numerical scheme, turbulence modelling, RANS, LES, DNS, deterministic inlet turbulence, jet-in-cross flow, shock-wave, shock trains, mixing enhancement and combustion mechanism. 